\title{
Gating Properties of Acetylcholine Receptors at Developing Rat Endplates ${ }^{1}$
}

\author{
STEFANO VICINI* AND STEPHEN M. SCHUETZE $\neq^{2}$ \\ *Fidia Research Laboratories, 35031 Abano Terme, Italy and $\ddagger$ Columbia University, Department of Biological Sciences, Fairchild \\ Center 913B, New York, New York 10027
}

\begin{abstract}
The gating properties of acetylcholine receptors (AChRs) change during the development of rat soleus endplates. During the first 3 weeks after birth, the apparent mean channel open time $(\tau)$ decreases severalfold and the singlechannel conductance $(\gamma)$ increases $50 \%$. To better understand this phenomenon, we used a combination of noise analysis, analysis of miniature endplate currents (MEPCs), and single-channel recordings to quantify the relative levels of fast and slow AChR activity at developing soleus endplates. When the same endplates were studied with both noise analysis and MEPC analysis, results obtained with the two techniques were strongly correlated, but MEPC analysis yielded higher estimates of the relative amount of slow channel activity. Experiments designed to examine the distribution of fast and slow channels gave no evidence for a gradient of either channel type within individual endplates; rather, fast and slow channels appeared to be mixed together. However, the relative amount of fast and slow channel activity did vary markedly among different endplates within individual muscles. In agreement with earlier studies, we found a progressive decrease in the relative amount of slow channel activity during the first 3 weeks after birth. However, our data indicate that this process begins sooner than reported previously and takes longer to complete. Some of the same endplates that were studied physiologically were also examined in the electron microscope to test the hypothesis that changes in AChR gating might be related to ultrastructural changes such as the formation of folds. The physiological and ultrastructural results were essentially uncorrelated.
\end{abstract}

Acetylcholine receptors (AChRs) undergo a number of changes during the development of skeletal muscle endplates (reviewed by Fambrough, 1979). In rats, these changes begin soon after the initial nerve-muscle contact and continue for the next 3 to 4 weeks, paralleling endplate development as a whole. The first wave of changes occurs prenatally during the third week of gestation when AChRs cluster beneath nerve terminals (Bevan and Steinbach, 1977), undergo a decrease in their metabolic turnover rate (Reiness and Weinberg, 1981; Steinbach, 1981), and show a shift in their

Received September 27, 1984; Revised January 8, 1985;

Accepted January 14, 1985

\footnotetext{
${ }^{1}$ We thank Drs. R. Pollock and L. Rubin for helpful discussions and Ms B. Moss for technical assistance. This work was supported by grants from the National Institutes of Health, the Muscular Dystrophy Association, and the March of Dimes. S. M. S. is a Sloan Research Fellow.

${ }^{2}$ To whom correspondence should be addressed.
}

isoelectric focusing point (Brockes and Hall, 1975). In a second, postnatal group of events, AChRs change in their response to curare (Ziskind and Dennis, 1978), in their expression of certain antigenic determinants (Reiness and Hall, 1981; Hall et al., 1984), and in their ionic gating properties (Sakmann and Brenner, 1978; Schuetze and Fischbach, 1978; Fischbach and Schuetze, 1980).

The most striking developmental change in $\mathrm{AChR}$ function is a 3- to 5-fold decrease in the apparent mean channel open time $(\tau$; Sakmann and Brenner, 1978; Schuetze and Fischbach, 1978; Fisch. bach and Schuetze, 1980), which is accompanied by a $50 \%$ increase in single-channel conductance $(\gamma$; Hamill and Sakmann, 1981; Siegelbaum et al., 1984). In rat soleus muscles, the decrease in $\tau$ begins a few days after birth and takes about 3 weeks to complete. During the transition period, endplates contain substantial numbers of both embryonic-type channels with long $\tau$ 's and adulttype channels with short $\tau$ 's.

Because the decay phase of synaptic currents directly reflects the closing of acetylcholine (ACh)-sensitive channels (Magleby and Stevens, 1972; Anderson and Stevens, 1973), the shape of synaptic currents also changes during development. Miniature endplate currents (MEPCs) decay several times more slowly in newborn endplates than in adult endplates (Sakmann and Brenner, 1978; Fischbach and Schuetze, 1980). During the postnatal transition period when fast and slow channels are present in the same endplates, MEPC decays are biphasic, consisting of an initial rapid phase followed by a second slower phase. The relative amplitudes of the two components presumably reflect the relative amounts of fast and slow channel activity (Michler and Sakmann, 1980).

The mechanism behind the $\tau$ decrease has not been elucidated, but it probably does not involve replacing the entire AChR, as the turnover rate of AChRs appears to be much slower than the changes in channel properties (Michler and Sakmann, 1980; Reiness and Weinberg, 1981). One possibility is that the changes in channel properties might be related to the formation of postsynaptic membrane folds (Fischbach and Schuetze, 1980; Brenner and Sakmann, 1983). Since both processes take place at the same time (Korneliussen and Jansen, 1976; Rosenthal and Taraskevich, 1977), it is conceivable that the segregation of AChRs to the tips of newly forming folds (Matthews-Bellinger and Salpeter, 1983) is associated with changes in channel gating.

To investigate further the developmental changes in endplate channel properties, we have used a combination of MEPC analysis, noise analysis, and single-channel recording to study AChR gating at more than 300 developing rat soleus endplates. Our results show that the $\tau$ decrease occurs over a longer period than reported previously and emphasize that different fibers within individual muscles do not undergo a synchronous $\tau$ decrease. In addition, it appears that fast and slow channels are not segregated from one another but, rather, are mixed together.

Some of the endplates that were studied with MtPC analysis were also examined in the electron microscope to test the hypothesis that 
the changes in channel properties might be related to the appearance of postsynaptic folds. When the exact same cells were char acterized physiologically and ultrastructurally, there was at most only a weak correlation between the extent of synaptic membrane folding and the relative amount of fast and slow channel activity estimated from the MEPCs.

\section{Materials and Methods}

Electrophysiology. All experiments were performed on the soleus muscles of Sprague-Dawley rats (Charles River Breeding Laboratories) 1 to 30 days after birth. Muscles were dissected free of surrounding tissue, pinned to a layer of cured Sylgard resin, bathed in well oxygenated L-15 tissue culture medium (Grand Island Biological Co., GIBCO), and placed on the stage of a Zeiss UEM microscope equipped with differential interference contrast optics. Endplates were clearly visible at $\times 500$, and at the edges of the preparation individual fibers could be distinguished uniquely from their neighbors. All recordings were done at 19 to $24^{\circ} \mathrm{C}$.

Spontaneous MEPCs were recorded with a focal extracellular pipette, similar to a patch-clamp pipette (Hamill et al., 1981), that was pressed against the edge of an endplate (Schuetze, 1980). Pipettes were constructed from Boralex 100- $\mu$ l capillaries (Rochester Scientific Co.) with a Kopf $700 \mathrm{C}$ puller and were fire polished with an electrically heated, glass-coated, platinumiridium wire. The electrode was connected to a List LM EPC-5 current-tovoltage converter. The signal was fed directly into the analog-to-digital converter of a Digital Equipment Corporation (DCC) LSI 11/23 computer and sampled at $20 \mathrm{kHz}$. MEPCs were detected by waiting for the signal to cross a threshold set at several times the peak-to-peak base line noise level. Usually 20 to 30 MEPCs were collected from each endplate and analyzed immediately thereafter (see below). At some endplates more than 200 MEPCs were collected and analyzed (ct. Figs. 7 and 8).

ACh-induced current fluctuations for noise analysis experiments were recorded extracellularly with similar electrodes that were filled with $20 \mu \mathrm{M}$ ACh in L-15 (because of hydrolysis by synaptic acetylcholinesterase, the $A C h$ concentration at the receptors was undoubtedly lower). A net inward current accompanied by a severalfold increase in the fluctuations was recorded by pressing the electrode against the edge of the endplate. The signal was low-pass filtered at $900 \mathrm{~Hz}$ (8-pole Butterworth) and digitized at 2 $\mathrm{kHz}$ for 25 to $65 \mathrm{sec}$. The samples were stored on a Winchester disk drive. The resistance increase induced by pressing the electrode against the cell was noted, and a control record for a background spectrum was obtained by pressing the electrode against a piece of Sylgard (or against a cell insensitive to $\mathrm{ACh}$ ) to achieve the same resistance increase. Experimental records were edited prior to analysis to remove segments containing superimposed MEPCs. The power spectra of the edited experimental and control records were computed and used to generate the difference spectrum.

Single ACh-sensitive channel currents were measured using cell-attached patch recordings. In these experiments, a small region near the endplate band was stripped of connective tissue with a fine glass hook. Cleaning was continued until the selected regions appeared naked when viewed at $\times 500$ with differential interference contrast optics. In some experiments, the preparation was treated with $0.1 \%$ collagenase (Sigma type I) in well oxygenated $\mathrm{L}-15$ for 15 to $30 \mathrm{~min}$. In most cases, however, no enzyme treatment was used; similar results were observed with both proccdures. Pipcttes werc constructed as described above for MEPC recordings and were filled with 0.1 to $0.5 \mu \mathrm{M} A C h$ in L-15. In some experiments, rat Ringer solution was used instead of L-15. The Ringer solution was made according to the melhod of Dennis et al. (1981) except that the serum was omitted. Gigaohm seals ( 5 to 60 gigaohms) were obtained with conventional techniques (Hamill et al., 1981), and currents through cell-attached membrane patches were measured with a List LM EPC-5 current-to-voltage converter. The current output signal was connected to a low-pass Bessel filter $\left(f_{\mathrm{c}}=1\right.$ to $3 \mathrm{kHz}, 8$ pole) for conductance measurements and to a low-pass Butterworth filter $\left(f_{c}\right.$ $=900 \mathrm{~Hz}, 8$-pole) for spectral analysis. The single-channel currents were digitized at 2 to $10 \mathrm{kHz}$ and stored on a Winchester disk drive.

Data analysis. MEPCs were fitted either with a simple exponential curve or with the sum of two exponentials. In both cases, fits were performed using only that part of the decay phase that fell in the range of 10 to $90 \%$ of peak MEPC amplitude. The initial part of each MEPC decay was ignored because of rounding near the peak; the final part was ignored because the signal fell into the base line noise level.

In single exponential fits, each MEPC decay phase was fitted to the equation

$$
I(t)=I_{0} \operatorname{EXP}(-t / \tau)
$$

where $I$ is current, $t$ is time, $I_{0}$ is the current at zero time, and $\tau$ is the exponential time constant. The base line (zero current level) was defined to be the average value of the trace during the $2.5 \mathrm{msec}$ preceding the rising phase of the MEPC. Time zero for MEPC decay was defined to be the time of peak MEPC amplitude. Least squares regression of the decay phase to this equation yielded estimates of $\tau$ and $I_{0}$.

In double exponential fits, each MFPC decay phase was fitted to the equation

$$
I(t)=l_{f} \operatorname{EXP}\left(-t / \tau_{t}\right)+l_{s} \operatorname{EXP}\left(-t / \tau_{s}\right)
$$

where $I_{i}$ and $I_{s}$ are the amplitudes of the fast and slow components of MEPC decays at time zero and $\tau_{f}$ and $\tau_{s}$ are the corresponding time constants. The zero current and zero time points were defined as for single-exponential fits. The two time constants were selected manually. The fast $\tau$ was set betwcen 0.9 and $1.3 \mathrm{msec}$ and the slow $\tau$ was set between 4.5 and 7.5 $\mathrm{msec}$. The values chosen depended on the bath temperature, which ranged between 19 and $24^{\circ} \mathrm{C}$. These values were consistent with the $\tau$ 's determined Irorr power spectra of ACh-induced current fluctuations recorded at the same temperature. After the $\tau$ 's were selected, estimates of $I_{f}$ and $I_{s}$ were obtained by fitting the MEPC decay phase to the preceding equation by least squares regression. The procedure was adopted because it was sufficiently fast and efficient to permit rapid on-line analysis. Figure 1 is typical of the goodness of fit. In obviously doubly exponential MEPCs, the summed squared deviation in double-exponential fits was 3 to 5 times smaller than that obtained with single-exponential fits.

In noise analysis experiments, the difference spectrum was fitted with the sum of two Lorentzian curves or, when appropriate, with a single Lorentzian curve. Single Lorentzian curve fits were done using an iterative, nonlinear, weighted, least squares regression routine (Schuctzc, 1980). In double Lorentzian fits, the experimental spectrum was fitted to the equation

$$
S(w)=S_{f} /\left[1+\left(w / w_{f}\right)^{2}\right]+S_{s} /\left[1+\left(w / w_{s}\right)^{2}\right]
$$

where $S(w)$ is the value of the power spectrum at frequency $w, S_{f}$ is the amplitude of the fast spcctral component at zero frequency, and $S_{s}$ is the amplitude of the slow spectral component at zero frequency. (The symbol $w$ is used for frequency rather than $f$ to avoid confusion with the subscript for "last.") Irilial guesses for the two corresponding critical frequencies $w_{f}$ and $w_{s}$ were selected manually, and least squares regression was used to find the optimum values of $S_{f}$ and $S_{s}$. Then, in an iterative procedure, each critical trequency was adjusted in turn and the regression was repeated until the sum of the squared deviations was minimized (generally 2 to 5 times lower than that obtained with single Lorentzian curves). This yielded estimates of the two critical frequencies and the two spectral amplitudes. A $\tau$ was derived from each critical frequency $W_{c}$ through the relationship $\tau=1 /\left(2 \pi W_{c}\right)$.

Single-channel recordings were analyzed with a simple, computer-assisted procedure. Channel openings in Bessel-filtered records were identified by eye, and their mean amplitudes were measured directly. The apparent mean channel open times within single channol recordings were estimated by spectral analysis of Butterworth-filtered records. These spectra were calculated and analyzed as described above for noise analysis experiments. The only modification was that background speclra were not calculated from separate records, but from segments of experimental records in which there were no channel openings.

Estimates of the relative numbers of fast and slow channel openings. In interpreting MEPCs and noise spectra, we modeled AChR channel openings as rectangular current pulses of fixed amplitude but random, exponentially distributed durations (Anderson and Stevens, 1973). We assumed that there were two types of channels that had different conductances and different mean open times. Although this is a reasonable approximation, recent studies have shown that $A C h R$ channel openings are frequently interrupted by brief closures that give them a burst-like appearance (Colquhoun and Sakmann, 1981). This means that the $\tau$ 's we estimated from MEPCs or from noise spectra are better interpreted as the mean duration of a burst of channel openings rather than as the mean channel open time. To emphasize this fact, we refer to $\tau$ as the apparent mean channel open time.

We estimated the relative numbers of the two channels types that opened during an MEPC from the results of the double exponential curve fits. It was assumed that

$$
\begin{aligned}
& I_{f}=N_{f} \gamma\left(V_{m}-V_{\text {rev }, f}\right) \text { and } \\
& I_{s}=N_{s} \gamma_{s}\left(V_{m}-V_{\text {rev }, s}\right)
\end{aligned}
$$

where $N_{t}$ and $N_{s}$ are the numbers of fast and slow channels operi di lime $t$ $=0$ (the time of peak MEPC amplitude), $\gamma_{i}$ and $\gamma_{s}$ are the two single-channel 
A

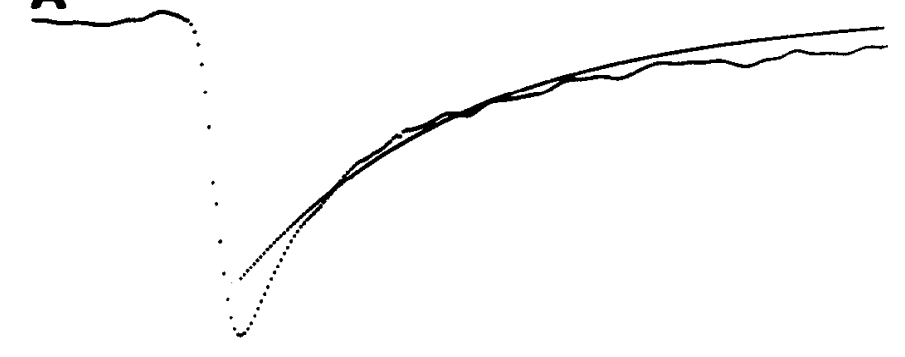

B
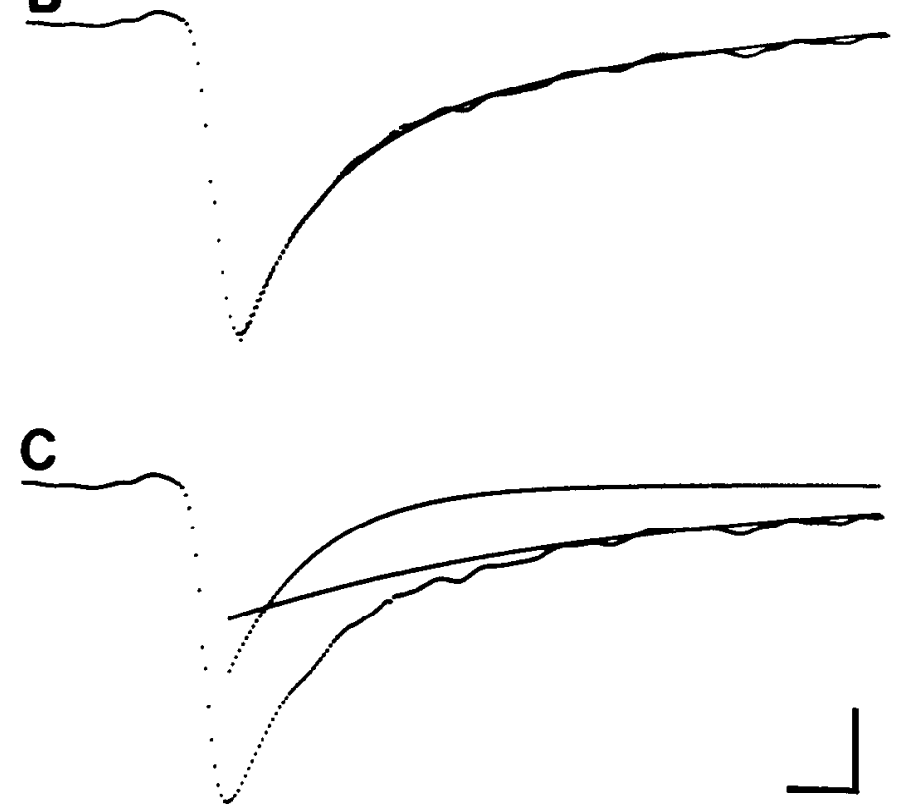

Figure 1. An MEPC fitted with one or two exponential curves. $A$, An MEPC recorded from a day 11 soleus endplate. The superimposcd simple exponential curve was fitted to its decay phase by least squares regression (time constant $=3.3 \mathrm{msec}$ ). $B$. The same MEPC fitted with the sum of two exponential curves with time constants of $6.2 \mathrm{msec}$ and $1.3 \mathrm{msec}$. $C$, The same MEPC with the two exponential components shown individually rather than summed as in $B$. At the time of peak MEPC current, the slow exponential was $41 \%$ of the total MEFC amplitude. Calibration bars $=50 \mathrm{pA}, 1 \mathrm{msec}$. Temperature $=21^{\circ} \mathrm{C}$

conductances, and $V_{\text {rev, } 1}$ and $V_{\text {rev,s }}$ are the reversal potentials for the two types of channels. Assuming that the two $V_{\text {rev }}$ 's are identical, it follows that

$$
N_{s} /\left(N_{s}+N_{i}\right)=I_{s} /\left[I_{s}+I_{i}\left(\gamma_{s} / \gamma_{i}\right)\right] .
$$

Based on our single-channel recordings, we assumed that $\gamma_{s} / \gamma_{t}$ was 0.67 .

Similar estimates of the relative numbers of fast and slow channel openings during noise recordings were obtained from the double Lorentzian fits to ACh power spectra. Here we assumed that (cf. Anderson and Stevens, 1973)

$$
\begin{aligned}
& S_{f}=N_{t} 4\left(\gamma_{f} \tau_{t}\right)^{2} \text { and } \\
& S_{s}=N_{s} 4\left(\gamma_{s} \tau_{s}\right)^{2}
\end{aligned}
$$

where $S_{1}$ and $S_{s}$ are the zero frequency asymptotes obtained from the

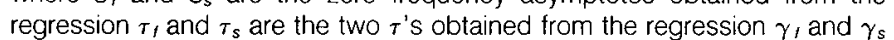
are the two single-channel conductances, and $N_{f}$ and $N_{s}$ are the frequencies at which the fast and slow channels opened (unitary bursts of openings per second) during the noise recording. From these equations it follows that

$$
N_{s} /\left(N_{s}+N_{t}\right)=S_{s} /\left[S_{s}+S_{f}\left(\gamma_{s} / \gamma_{f}\right)^{2}\left(\tau_{s} / \tau_{f}\right)^{2}\right] .
$$

As before, based on our single-channel recordings we assumed that $\gamma_{s} / \gamma_{r}$ was 0.67 . The validity of this analysis was tested experimentally ( $\mathrm{rig} .3$ ).

Generation of simulated MEPCS. In several experiments, MEPCs recorded from a developing endplate were compared with simulated MEPCs generated using a simple computer model (Fig. 7). The goal was to identify factors responsible for variations in the decay phases of MEPCs at individual endplates. The model was implemented on a DEC LSI 11/23 computer and was based on the following assumptions. (1) Each MEPC was due to the instantaneous, synchronous opening of $1000 \mathrm{ACh}$-sensitive channels at time $t=0$. No channel openings occurred after time $t=0$. The number 1000 was based on measurements of MEPC peak amplitudes reported by Michler and Sakmann (1980) and on our estimates of the conductances of fast and slow channcls. (2) Each of the 1000 channels that opened had one of two possible apparent mean open times $\left(\tau^{\prime} s\right)$. S percent of the 1000 had long $\tau$ 's $(\sim 6 \mathrm{msec})$, and the rest had brief $\tau$ 's $(\sim 1 \mathrm{msec})$. (3) Each channel opening generated a reclangular current pulse of fixed amplitude but with a random, exponentially distributed duration. (4) The single-channel currents of the faster channels were $50 \%$ greater than the currents through the slower channels (ct. Fig. 6). (5) The tast and slow channels were uniformly mixed, and ACh bound to AChRs randomly. Thus, when the quantum of neurallyreleased $A C h$ opened 1000 channels, the number of slow channels that opened was governed by a binomial distribution dependent on $S$.

The procedure for simulating an MEPC from any particular endplate was as follows. First, the parameters $S, \tau_{\varepsilon}$, and $\tau_{f}$ were set equal to the values measured experimentally. Second, the number of slow channels that opened $\left(N_{s}\right)$ was drawn from a binomial distribution in which $n=1000$ and $p=S /$ 100 . Third, $N_{s}$ square pulses of unit amplitude were constructed. The duration of each was drawn independently from an exponential distribution defined by $\tau_{s}$. Fourth, $\left(1000-N_{s}\right)$ square pulses were constructed. The amplitude of these was set at 1.5 units (reflecting the $50 \%$ greater conductance of lasl channels). The durations were drawn independently from an exponential distribution defined by $\tau_{f}$. Fifth, the rising phases of the 1000 square pulses were aligned and the pulses were summed. Finally, a nolse signal recorded under conditions similar to those of the original experiment was added to the simulated MEPC. The result was a function that appeared much like a recorded doubly exponential MEPC except that its rising phase was instantaneous.

Electron microscopy. Physiologically characterized cells were labeled using a modification of the Lucifer Yellow-diaminobenzidine technique of Maranto (1982) similar to that described by Schuetze and Vicini (1984). In brief, after a selected fiber was studied physiologically, it was labeled with an intracellular injection of Lucifer Yellow (Fig. 9). Then the preparation was fixed, washed, and bathed in a diaminobenzidine solution ( $1 \mathrm{mg} / \mathrm{ml}$ in L-15). The labeled cell was relocated in the light microscope, and a portion of the iabeled fiber adjacent to the endplate was illuminated with UV light for 15 to $45 \mathrm{~min}$. This generated an opaque marker in the illuminated region that was readily identifiable in the electron microscope and yet did not obscure the endplate. Cells were labeled only if the amount of fast channel activity in MEPCs was at least $30 \%$.

Morphometry was performed using a digital bit.pad connected to a simple microprocessor. The degree of postsynaptic folding was quantified by calculating a folding index defined as the ratio of the length of endplate membrane to the length of the endplate through the midline of the primary synaptic cleft (cf. Matthews-Bellinger and Salpeter, 1983).

\section{Results}

Quantitative comparison of MEPC decays and ACh noise spectra at developing endplates. Although MEPCs decay as simple exponentials at embryonic and adult rat endplates, at early postnatal endplates they decay as the sum of two exponential curves (Sakmann and Brenner, 1978; Fischbach and Schuetze, 1980) (Fig. 1). These doubly exponential decays arise from the dual population of AChRs present in developing endplates. The adult-like AChRs with short apparent mean channel open times ( $\tau^{\prime} s$ ) give rise to the initial rapid decay, and the embryonic-like channels with long $\tau$ 's give rise to the slow decay.

The relative amplitudes of the two exponential components of MEPC decays reflect the relative activities of the two types of channels that were activated by the neurally released ACh (Michler and Sakmann, 1980). This can be quantified by scaling the peak amplitude of the two components inversely by the appropriate singlechannel conductances (see "Materials and Methods" for details). The single-channel conductances were determined from patchclamp recordings (see below) that showed that the conductance of adult-type, fast channels is about $50 \%$ greater than that of embryonic-type, slow channels. As an example of this analysis, note that, 


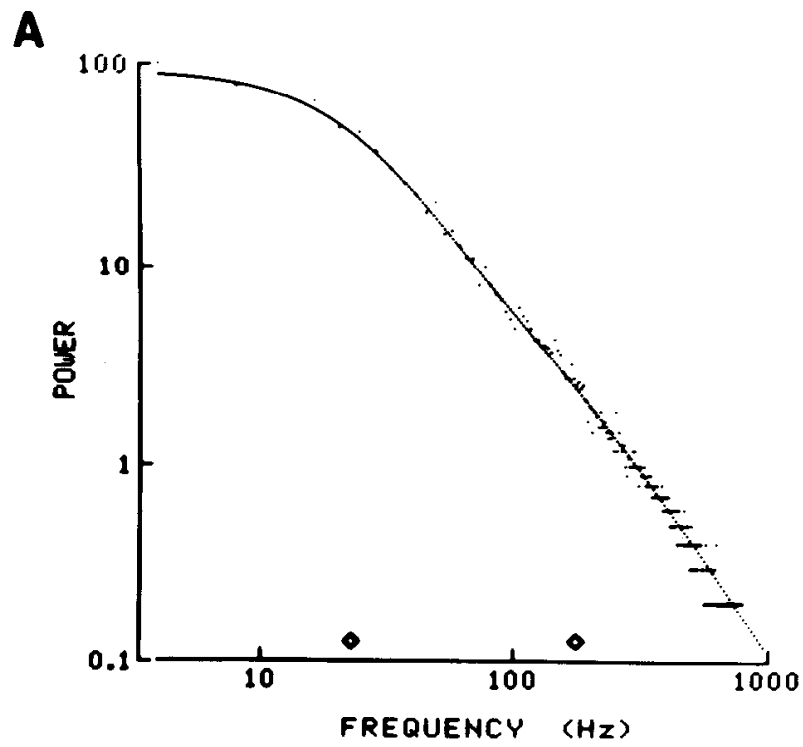

B

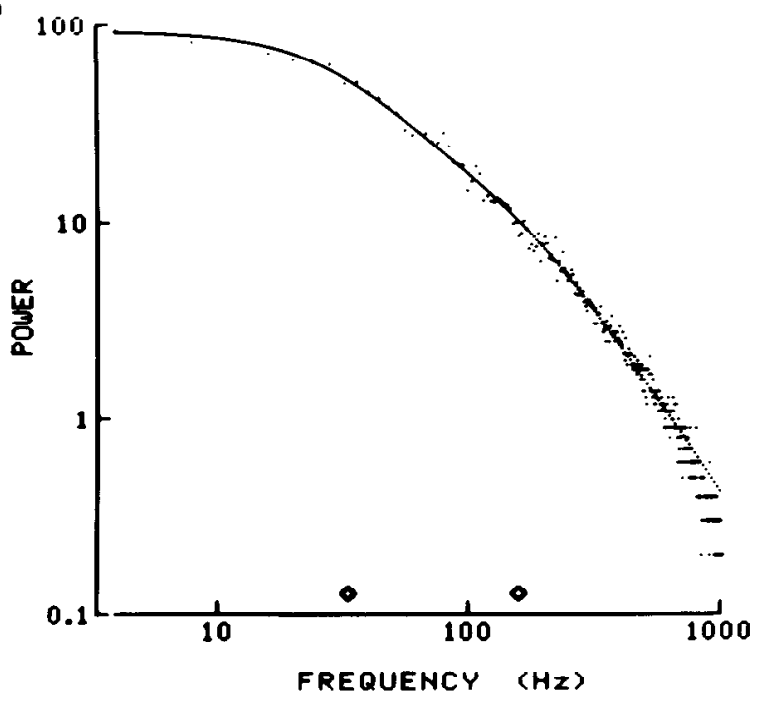

C

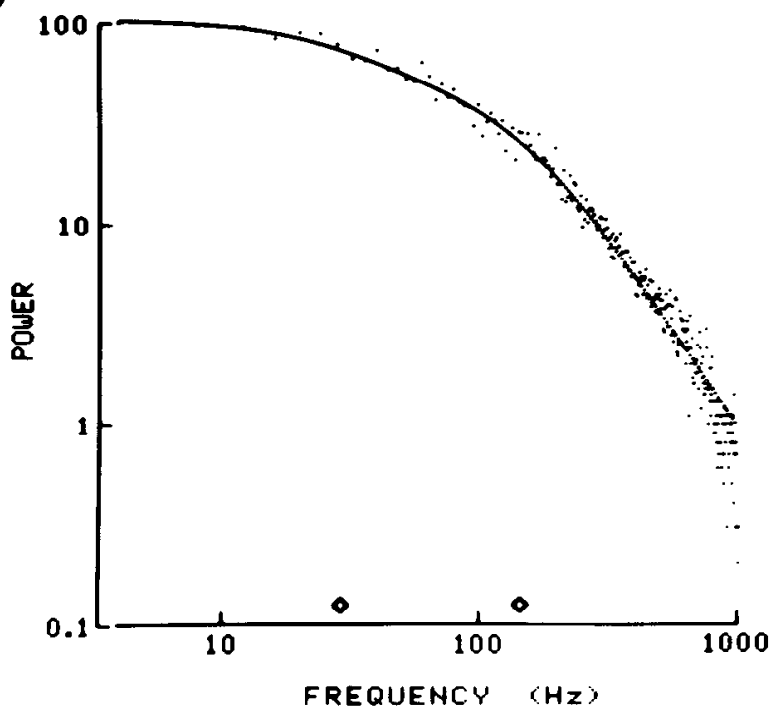

in the MEPC shown in Figure 1, the fast exponential component is $60 \%$ of total peak amplitude and the slow component is $40 \%$. That is, the fast component is $50 \%$ greater than the slow one. Taking into account the $50 \%$ grcater conductance of the fast channels, this indicates that roughly equal numbers of fast and slow channels were open during the peak of this MEPC.

As expected from earlier work (Sakmann and Brenner, 1978; Fischbach and Schuetze, 1980), we found that the power spectra of ACh-induced current fluctuations recorded from endplates with doubly exponential MEPCs were also complex. That is, unlike the single Lorentzian spectra found at adult and embryonic endplates, spectra at these developing endplates were best described by the sum of two Lorentzian curves, one for each of the two channel populations (Fig. 2). Just as the two components of MEPC decays reflect the relative activities of fast and slow channels in response to neurally released $\mathrm{ACh}$, the relative amplitudes of the two Lorentzian components of $\mathrm{ACh}$ power spectra should reflect the relative activities of fast and slow channels to exogenously supplied ACh. However, the relationship between fast and slow channel activities and $\mathrm{ACh}$ power spectra is complex and depends on the relative amplitudes of the two spectral components, the two $\tau$ 's, and the two single-channel conductances (see "Materials and Methods").

We tested the reliability of the spectral estimates of fast and slow channel activities by applying the procedure to single-channel recordings of AChRs in rat myotubes. Since the two channel types have different conductances, fast and slow channel openings give rise to events of different amplitudes that can be distinguished by inspection. Thus, direct counts of the relative numbers of high and low conductance events can be compared with the relative amount of fast and slow channel activity estimated by spectral analysis. Tissue-cultured myotubes were used instead of soleus muscles for these experiments because of the greater frequency of channel openings and the greater ease of obtaining gigaohm seals.

The percentages of high and low conductance channel openings estimated by direct counts were strongly correlated with the percentages of fast and slow channel openings estimated by spectral analysis of the same record (Fig. 3). Our estimate of the percentage of low conductance channel openings in the record shown partially in Figure $3 A$ was $78 \%$. This estimate was rather crude, for we ignored events that were too small to be assigned unambiguously to either category; these presumably were very brief openings that were attenuated by the filter. Nevertheless, the percentage of slow channel openings estimated from the power spectrum of the same record was nearly identical $(80 \%)$. In a total of 22 such experiments in which the percentage of slow channel openings ranged from $59 \%$ to $99 \%$, the mean difference between the two estimates (percentage of slow channcls - pcrcentage of low conductance channels) was $1.9 \% \pm 5.4 \%$ (mean $\pm \mathrm{SD})$.

This approach should be equally reliable in analyzing ACh noise recordings. In fact, repeated measurements made on the same endplates showed that the reproducibility of the technique was good. In the most complete experiment, nine noise records were obtained from the same endplate. The results of the nine analyses

Figure 2. ACh noise spectra from three soleus endplates with doubly exponential MEPCs. A, Spectrum from a day 8 endplate at which the slow MEPC component was $80 \%$ of total MEPC amplitude. Superimposed on the spectrum is the best fit double Lorentzian curve. The $\tau$ 's derived from the two Lorentzians were 7.0 and $0.9 \mathrm{msec}$. At zero frequency the ratio of the slow Lorentzian component to the the fast Lorentzian component was 41. The relative amplitudes of the two Lorentzian components are not simply related to the amplitudes of the two MEPC components (see the text). $B$, Spectrum from a day 9 endplate at which the slow MEPC component was $32 \%$ of total MEPC amplitude. The $\tau$ 's derived from the Lorentzians were 4.8 and $1.0 \mathrm{msec}$; the Lorentzian component ratio at zero frequency was 5.3. C. Spectrum from a day 14 endplate at which the slow MEPC component was $8 \%$ of total MEPC amplitude. The spectral $\tau$ 's were 5.5 and $1.1 \mathrm{msec}$; the Lorentzian component ratio was 1.1. In $A$ to $C$ the diamonds indicate the critical frequencies of the two Lorentzians. Temperature $=20-21^{\circ} \mathrm{C}$. 
A

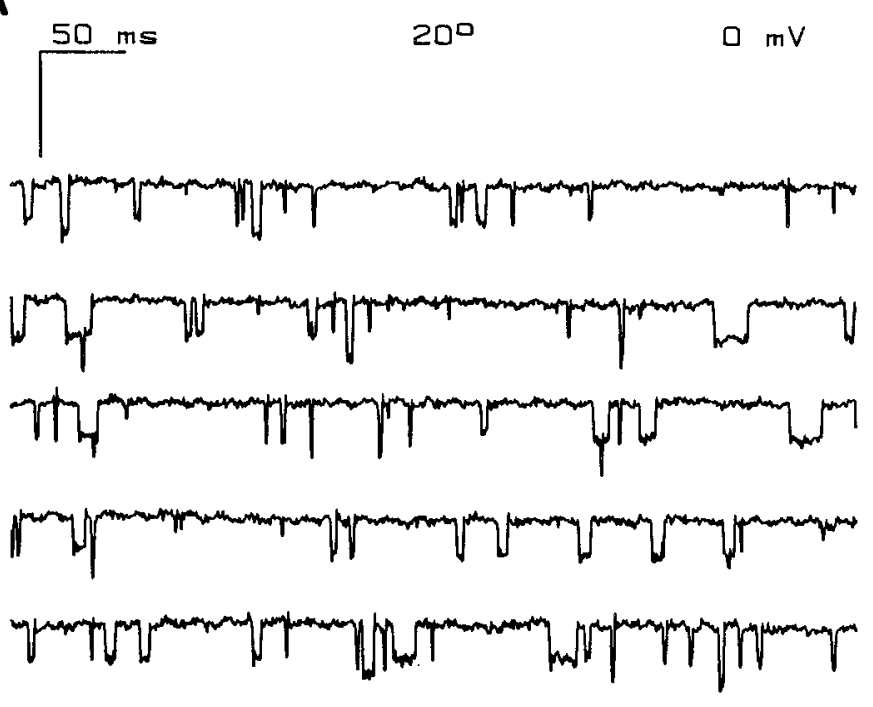

B

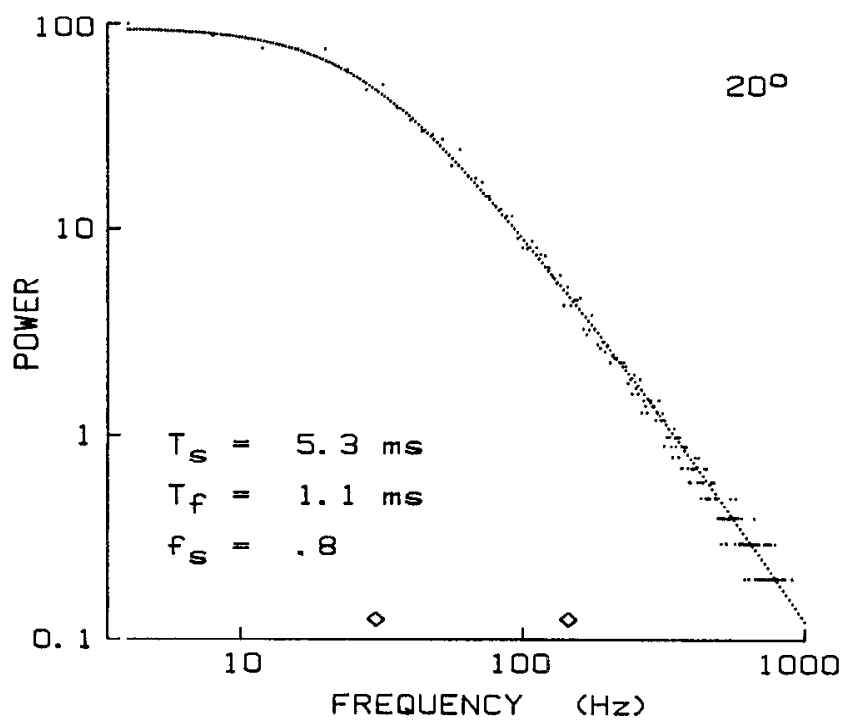

Figure 3. Spectral analysis of a cell-attached patch clamp recording from a rat myotube grown in tissue culture (4 days after plating). $A$, Nonconsecutive segments of a record of channel openings induced by $150 \mathrm{nM} \mathrm{ACh}$. The recording was obtained at the normal resting potential of the cell and was low-pass filtered at $900 \mathrm{~Hz}$ (8-pole Butterworth). Note that the currents generally fell into one of two amplitude categories, except for brief events that were attenuated by the filter and could not be categorized. It was estimated that $78 \%$ of the events fell into the lower amplitude category. Vertical bar $=7.5 \mathrm{pA}$. B. Spectrum of the entire record represented in $A$ with the best fit double Lorentzian curve superimposed. The diamonds indicate the two corner frequencies and correspond to $\tau$ 's of 5.3 and $1.1 \mathrm{msec}$. It was estimated from this spectrum that $80 \%$ of the channel activity in the original record was contributed by events associated with the slower Lorentzian component.

yielded estimates ranging from $28 \%$ to $41 \%$ slow channel openings; the mean was $35 \%$ and the standard deviation was $5.2 \%$.

Paired MEPC and noise recordings made at 52 developing soleus endplates showed a strong correlation between the relative amounts of slow channel activity in MEPCs and in noise records (Fig. 4). This suggests that the relative amplitudes of fast and slow decay phases of MEPCs is a useful measure of the relative amounts of fast and slow channel activity at developing endplates. However, although the two techniques agreed qualitatively, they disagreed quantitatively. The percentage of slow channel openings estimated from
MEPCs was about $60 \%$ greater than estimates determined from noise records from the same cells. We believe that much of this discrepancy reflected a systematic underestimation of fast channel activity by our MEPC analysis procedure, because fast channels that closed during the MEPC rising phase were ignored (see "Discussion").

Developmental decrease in the percentage of slow channel activity. The relative amount of slow channel activity was estimated from MEPCs recorded at 282 endplates and from noisc spectra recorded at 77 endplates (Fig. 5). The results show that some fast channel activity can be detected as soon as 1 to 2 days after birth, and that some slow channel activity persists for at least 3 weeks after birth. Thus, the developmental changes in channel gating do not have an abrupt onset or offset. It may be that some slow channels persist even into adulthood. Both MEPCs and spectra indicated $>20 \%$ slow channel activity at some endplates in 28 to 30-day-old rats (data not shown), but we did not study older animals.

Several other feature were noted in these experiments. First, although the relative amounts of fast and slow channel activity changed markedly with age, the $\tau$ 's of the two channel populations changed little (Table I). Thus, the kinetics of each channel population appeared to be constant throughout development. Second, in agreement with the results described above (Fig. 4), the percentage of slow channel activity estimated from MEPCs tended to be higher than that estimated from noise spectra. Third, the relative amount of slow channel activity at each age varied considerably from endplate to endplate. This was equaliy true for different endplates in an individual muscle and for endplates in different muscles of the same age. Furthermore, within individual muscles, neighboring fibers were no more similar than fibers spaced further apart. Thus, there was no evidence for a gradient of endplate maturity within developing muscles.

Single-channel recordings from developing soleus muscles. Es timating the relative number of slow channel openings from MEPCs and spectra required values for the unitary conductances of the two AChR populations. These were obtained from single-channel recordings of AChRs in perijunctional regions of developing soleus endplates (Fig. 6, Table II). The majority of the recordings were performed using the cell-attached patch configuration.

In every case studied, we found two populations of events that differed about $50 \%$ in amplitude. The records were similar to those obtained from rat myotubes in tissue culture, except that in soleus the event frequency was generally much lower and the fraction of large events was occasionally much higher, especially in older muscles. Although we did not do a detailed analysis of the singlechannel recordings, it is clear that the lower conductance events were due to the slow, embryonic-typc channels and the higher conductance events were due to fast, adult-type channels. In young muscles ( $<4$ days), high conductance channels were relatively rare and spectra of the single-channel recordings yielded long $\tau$ 's (6 to $9 \mathrm{msec}$ ). In older muscles (>18 days), low conductance events were rare and the spectra yielded short $\tau$ 's (1 to $2 \mathrm{msec}$ ). This interpretation is in agreement with the results of others (Siegelbaum et al., 1984).

Both types of channels appeared to rectify at hyperpolarized potentials (Table II). This was more pronounced when the patch pipettes were filled with $A C h$ dissolved in $\mathrm{L} 15$ rather than in rat Ringer (data not shown). One possibility is that the rectification was due to the arginine in the $\mathrm{L}-15$ medium (cf. Sanchez et al., 1983), but we did not explore this further.

A more detailed examination of doubly exponential MEPCs. When different biphasic MEPCs from the same endplatc worc fitted with the sum of two exponential curves, the relative size of the slow component varied from one MEPC to the next. Therefore, we normally recorded and analyzed 20 to 30 MEPCs at each endplate and averaged the results. This reduced the standard error of the mean of the percentage of slow channel openings to about $\pm 2 \%$. To understand better the origins of this variability, however, in seven 


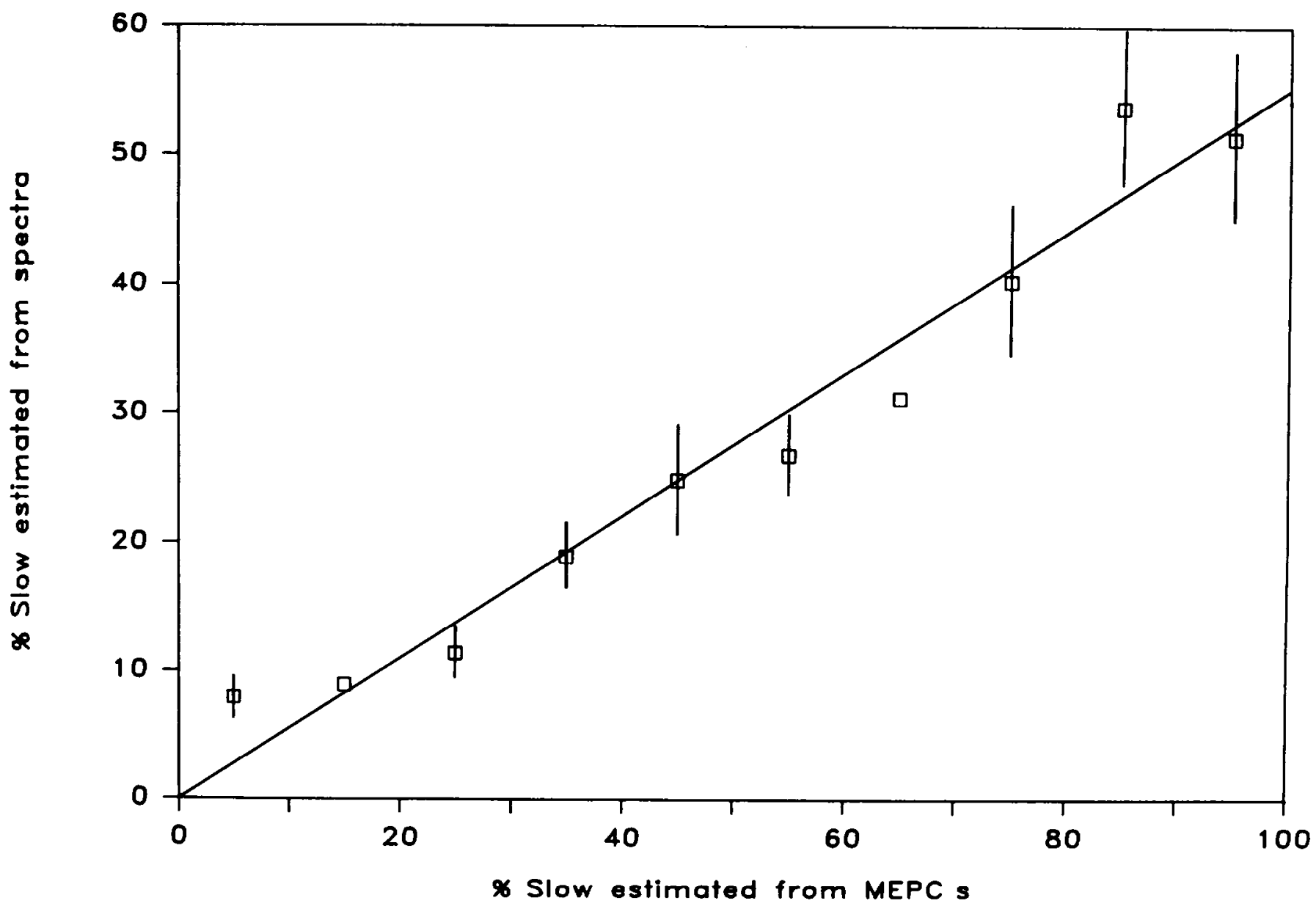

Figure 4. A comparison of the relative amount of slow channel activity during ACh-noise recordings and during MEPCs at the same soleus endplates. Both ACh noise and MEPCs were recorded from the same 52 soleus endplates and analyzed to determine the relative amount of slow channel activity in each case. Based on the results of the MEPC analysis, the data were pooled into bins of width $10 \%$ and averaged. The bars indicate \pm 1 SEM for bins containing three or more pairs of measurements. The line was fitted to the data by least squares regression.

experiments we collected and analyzed >200 MEPCs at individual endplates.

The results of one experiment based on 203 MEPCs recorded from the same endplate are illustrated in Figure 7. The slow exponential component averaged $35 \%$ of the total peak MEPC amplitude, but in one-third of the MEPCs the slow component was either $<30 \%$ or $>40 \%$ of total amplitude. The results were similar at the six other endplates studied.

One explanation is that, even within a single endplate, the relative numbers of fast and slow channels may vary from one site to the next. That is, different quanta of ACh may "see" different proportions of the two types of channels. We tested this by using the amplitude of extracellularly recorded MEPCs to indicate the relative position of the transmitter release site. All other factors being equal, the peak amplitude of the MEPC should indicate the distance between the active site and the recording electrode (cf. Fig. 5 in Frank and Fischbach, 1979).

When the relative size of the slow component was plotted against peak MEPC amplitude, there was no correlation between the two variables (Fig. 8). This was true at all seven endplates tested, indicating that there is no gradient of either channel type across an endplate. However, these results do not rule out the possibility of some microheterogeneity in channel distribution, such as small islands enriched for either fast or slow channels.

Alternatively, it may be that the observed variance in doubly exponential MEPCs is an intrinsic property and should be expected even if both channel types were mixed homogeneously. We tested this by using a simple mathematical model to generate simulated
MEPCs that took into account three sources of variance (see "Materials and Methods" for details): (1) variations in the number of slow and fast channels activated from trial to trial due to the random binding of $\mathrm{ACh},(2)$ the random, exponentially distributed durations of individual channel openings, and (3) noise superimposed on MEPCs by the recording apparatus. The simulated MEPCs were analyzed in the same way as those recorded experimentally.

The results showed that the simulation could account for about $75 \%$ of the observed variance in the estimates of percentage of slow channel activity (Fig. 7). Further analysis indicated that about $10 \%$ of the variance arose from trial-to-trial variability in the proportion of slow channel activation, about $25 \%$ was due to the random duration of channel openings, and about $40 \%$ was due to equipment noise (data not shown). Possible sources of the remaining $25 \%$ of the variance are given under "Discussion." We conclude that MEPC decays provide no evidence for heterogeneity in the distributions of fast and slow channels within an endplate.

The ultrastructure of endplates with different levels of slow channel activity. It has been suggested that the developmental decrease in $\tau$ might be related to the formation of postsynaptic membrane folds (Fischbach and Schuetze, 1980; Brenner and Sakmann, 1983). Both events happen during the first three postnatal weeks (Korneliussen and Jansen, 1976; Rosenthal and Taraskevich, 1977), and the formation of folds is accompanied by a segregation of AChRs to the tips of the folds (Matthews-Bellinger and Salpeter, 1983). To test this proposal directly, we used MEPC analysis to estimate the relative numbers of fast and slow channels at nine different soleus endplates. These same nine fibers were labeled with an electrondense marker (Fig. 9) and examined in the electron microscope. 


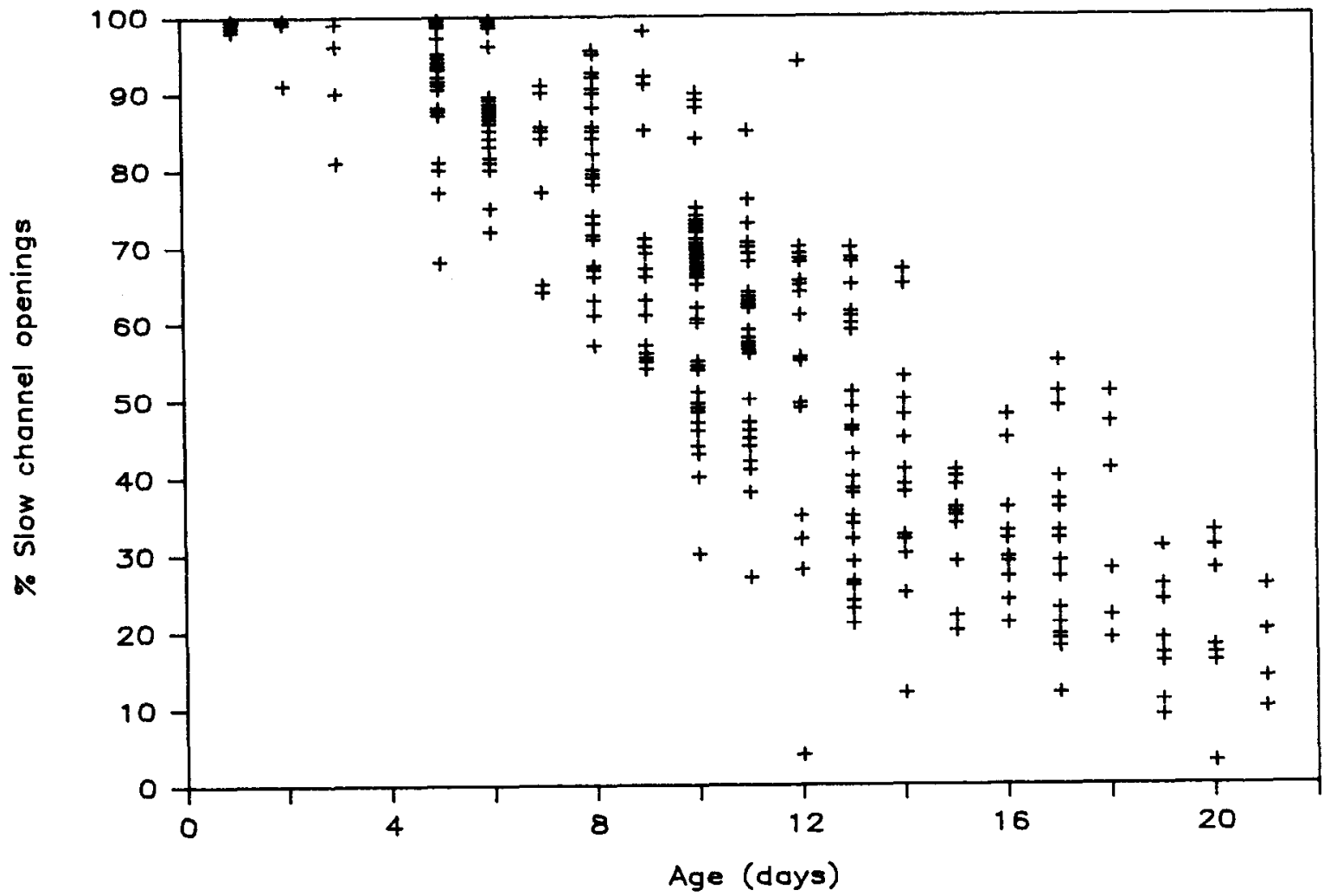

B

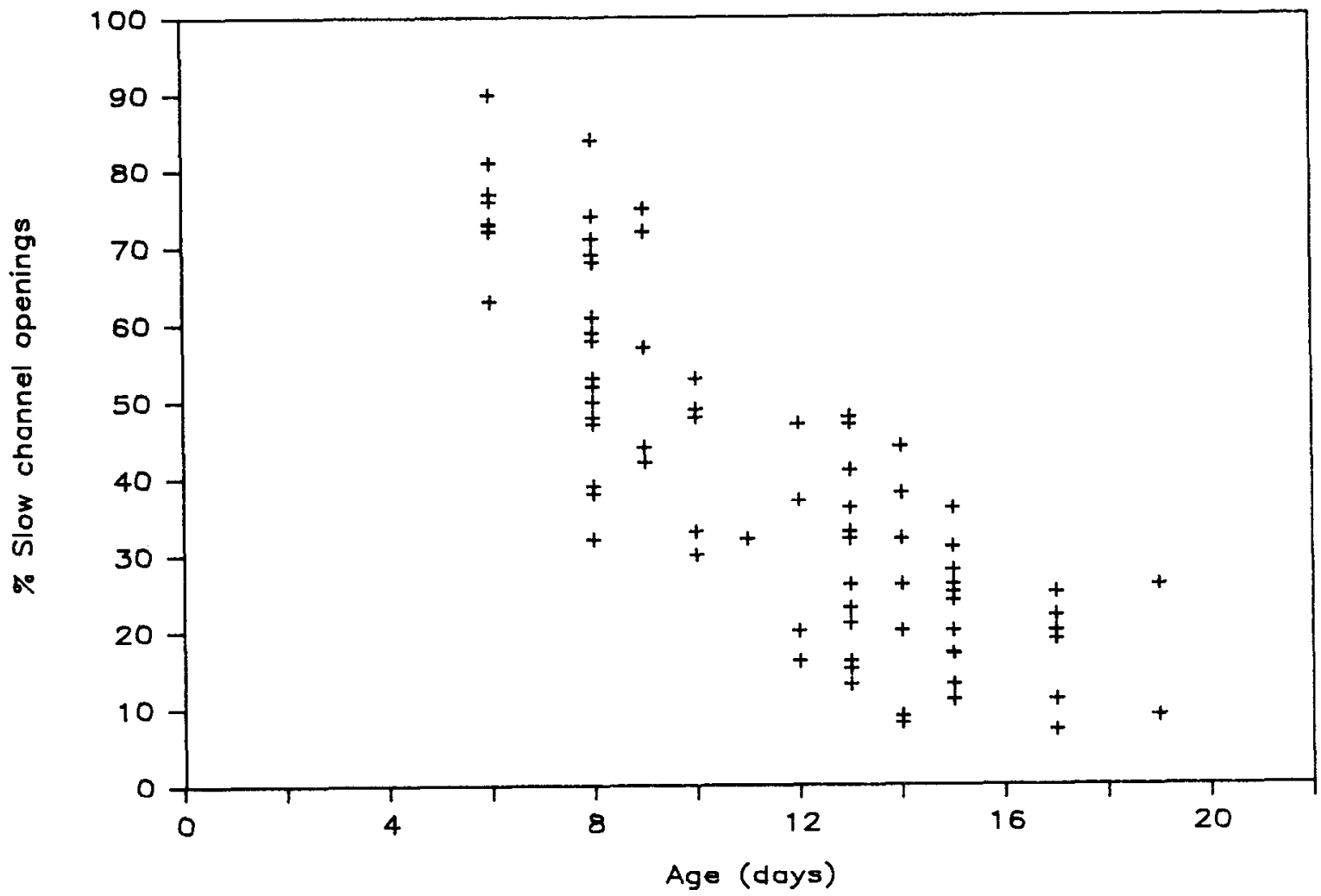


TABLE I

Tau at different ages

\begin{tabular}{|c|c|c|c|c|c|c|}
\hline \multirow{2}{*}{ Age } & \multicolumn{3}{|c|}{ Fast $\tau$ (msec) } & \multicolumn{3}{|c|}{ Slow $\tau$ (msec) } \\
\hline & Mean & SD & $N^{a}$ & Mean & SD & $N$ \\
\hline Postnatal days $6-10$ & 1.23 & 0.18 & 34 & 6.97 & 1.18 & 34 \\
\hline Postnatal days $11-19$ & 1.14 & 0.25 & 43 & 6.40 & 1.09 & 43 \\
\hline
\end{tabular}

${ }^{a} \mathrm{~N}$, number of cells tested. All values were derived from ACh noise spectra.
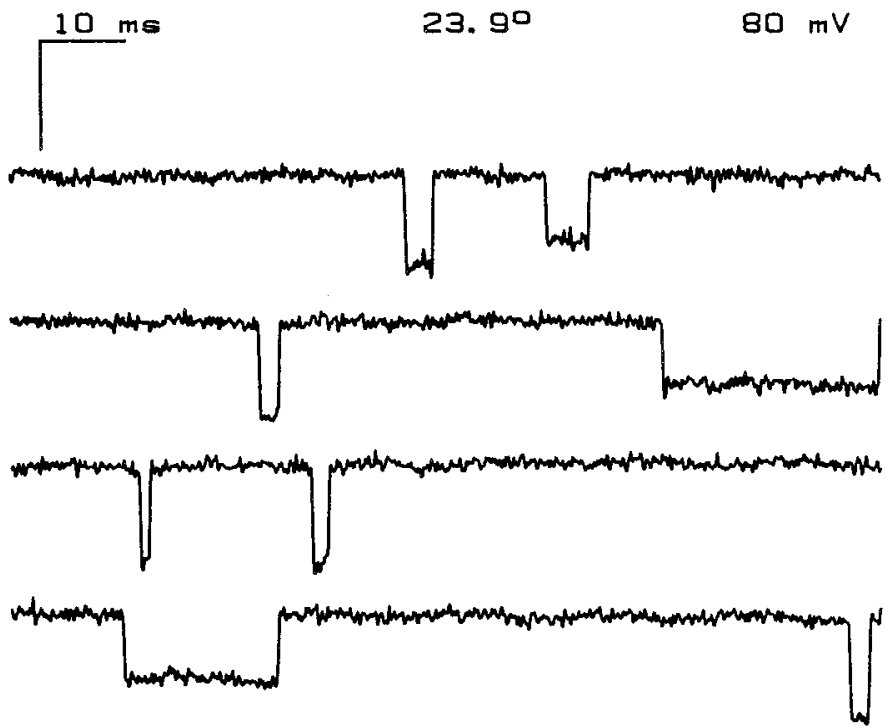

Figure 6. Nonconsecutive records of single-channel activity induced by $500 \mathrm{~nm}$ ACh at a perijunctional site on a day 9 soleus muscle fiber. Note that the currents fell into two amplitude catcgorics. The recording was obtained with the membrane hyperpolarized by $80 \mathrm{mV}$ from rest and was low-pass filtered at $2 \mathrm{kHz}$ (8-pole Bessel). No enzyme treatment was used. Vertical bar $=7.5 \mathrm{pA}$.

\section{TABLE ॥}

Single-channel conductance at different membrane potentials

Channel conductances were determined from the amplitudes of AChinduced channel openings recorded with cell-attached patch pipcttes. For each cell, $\sim 30$ to 100 events were measured from one patch and the results were broken into large and small groups. The two groups usually did not overlap except when the pipette potential was $0 \mathrm{mV}$, in which case some events were ambiguous and were discarded. Conductances at the normal resting potential were determined by dividing the current amplitudes measured at a pipette potential of $0 \mathrm{mV}$ by an assumed driving potential of -75 $m V$. For each of the other two potential ranges, events were measured at two pipette potentials $(0 \mathrm{mV}$ and either 40 or $80 \mathrm{mV})$ and the difference in current amplitudes was divided by the difference in pipette potentials.

\begin{tabular}{|c|c|c|c|c|c|c|}
\hline \multirow{3}{*}{$\begin{array}{c}\text { Pipette } \\
\text { Potentials } \\
\text { (mv) }\end{array}$} & \multicolumn{6}{|c|}{ Charinel Conductance (pS) } \\
\hline & \multicolumn{3}{|c|}{$\begin{array}{l}\text { High Conductance } \\
\text { Channel }\end{array}$} & \multicolumn{3}{|c|}{$\begin{array}{c}\text { Low Conductance } \\
\text { Channel }\end{array}$} \\
\hline & Mean & SD & $N^{a}$ & Mean & SD & N \\
\hline 0 & 48.3 & 3.2 & 15 & 34.6 & 2.0 & 15 \\
\hline 0 and +40 & 37.0 & 4.0 & 10 & 25.8 & 1.4 & 9 \\
\hline 0 and +80 & 32.5 & 5.3 & 8 & 22.7 & 3.0 & 8 \\
\hline
\end{tabular}

${ }^{a} N$, number of cells tested.

When postsynaptic folding at the labeled endplate was quantified by morphometry, we found no striking correlation between the extent of folding and the relative size of the slow MEPC component (Figs. 10 and 11). In the example shown in Figure 10, there were virtually no postsynaptic folds in an endplate at which the fast exponentia decay component was $41 \%$ of the peak MEPC amplitude. As shown in Figure 11, the formation of postsynaptic folds and the $\tau$ decrease appeared to be correlated only to the extent that both processes tended to advance with the age of the animal. Thus, these two processes do not appear to be directly related.

\section{Discussion}

Quantifying the relative amount of slow and fast channel activity These experiments differ from earlier developmental studies of AChR gating in rat muscle in several ways. First, a systematic effort was made to quantify the relative amounts of fast and slow channel activity at each endplate studied. Second, in many cases MEPCs and noise spectra were recorded from the same endplates so that the two techniques could be compared directly. Third, some of the physiologically characterized endplates were studied in the electron microscope, permitting a direct comparison of functional and structural characteristics. Finally, the number of endplates studied was large enough $(>300)$ to give a more complete picture of the time course of channel conversion.

Several measures helped to minimize the variance in our estimates of the relative levels of fast and slow channel activity. A major factor was maximizing the signal amplitude by using extracellular electrodes with relatively small tips $(\sim 1 \mu \mathrm{m}$ internal diameter). These were more effective than larger electrodes, probably because the tips could probe deeper into the endplate region. When the optics were favorable, it appeared that the largest signals were obtained when the pipette tip was positioned between the muscle surface membrane and an overlying cell, presumably a Schwann cell.

To take full advantage of small electrodes, it was helpful to perform extracellular recordings using a current-to-voltage converter, such as a patch clamp amplifier, rather than an electrometer. With an electrometer the high resistance of the small electrode tips decreased the frequency response and increased the noise level of the recordings. With a current-to-voltage converter, however, the frequency response was essentially independent of resistance and the noise level decreased when high resistance electrodes were used. The other major factor in minimizing variance was the use of long records. ACh noise spectra recordings were 25 to $65 \mathrm{sec}$ long, which yielded slender spectra. Similarly, estimates from MEPCs were improved by analyzing 20 to 30 events and averaging the results.

One consequence of the large signals and improved frequency response obtained in these experiments was that the fast and slow components could be separated more cleanly than in our previous studies (Fischbach and Schuetze, 1980). We found that the fast and slow $\tau$ 's differed by a factor of more than 5 rather than 3 or 4 as reported earlier (Fischbach and Schuetze, 1980). In 77 spectra, the mean ratio of $\tau_{\text {slow }}$ to $\tau_{\text {fast }}$ was $5.7 \pm 0.14$ (SEM).

The high signal amplitudes and the large difference between the two $\tau$ 's made it easier to estimate the relative amounts of fast and slow channel activity in MEPCs and in noise spectra. When relatively large, low noise signals were obtained, the sum of the squared deviations with double component curve fits was usually at least 2 fold lower than with single fits. This was true even when the activity of the minor channel population was as little as 10 to $15 \%$ of the total. When there were roughly equal levels of fast and slow channel activity, the double fits were often more than 5 -fold better than single fits.

Analysis of MEPC decays is an especially rapid, simple technique for estimating the relative amounts of fast and slow AChR activity at developing endplates. Extracellular MEPC recordings are technically easier and less likely to damage the endplate than noise recordings, probably because the electrode position is less critical. In addition, the analysis of MCPCs, once automated, is very fast and easily performed on line with a laboratory computer. This makes the technique especially suitable for following individual endplates over time (S. M. Schuetze and S. Vicini, manuscript in preparation) and before and after treatment with antibodies or other reagents (cf. Schuetze et al., 1985). MEPC analysis appears to be reliable in that, at any particular endplate, the amount of slow channel activity 


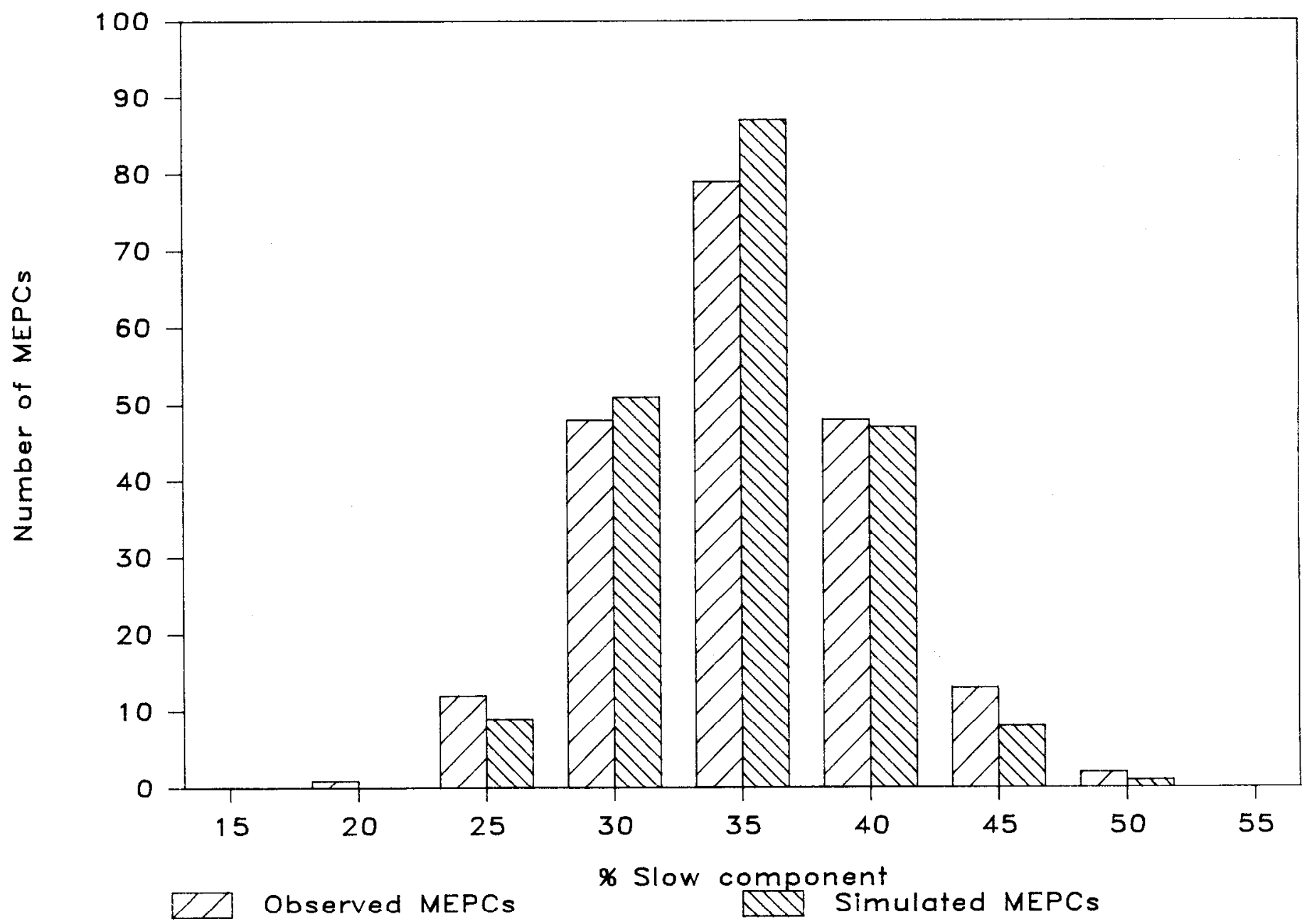

Figure 7. Variability in MEPC decays at the same endplate. Two hundred three MEPCs were recorded from a day 11 endplate and fitted with doubly exponential curves. The histogram shows the distribution of the amplitude of the slow exponential component expressed as a fraction of total MEPC amplitude (mean $=35 \%$ slow). An equal number of simulated MEPCs with the same mean level of slow channel activity were generated with a simple computer model (see the text), and both sets of MEPCs were analyzed with the same procedure. The standard deviations in these distributions were $5.1 \%$ (observed) and $4.4 \%$ (simulatcd), indicating that the model accounts for about $75 \%$ of the variance.

estimated from MEPCs is strongly correlated with estimates derived trom noise spectra.

One limitation of this technique, however, is that MEPC analysis overestimates slow channel activity compared to the more direct approach of noise analysis. At least in part, this discrepancy arose because the MEPC analyses estimated the relative numbers of fast and slow channels that were open during the MEPC peak. Channel openings that occurred during the MEPC rising phase were not taken into account. Both fast and slow channels were opening and closing during each MEPC rising phase, but by their very nature, fast channels were the more likely to close before the peak. Thus, our MEPC analysis inevitably underestimated the number of fast channel openings that occurred over the entire time course of the MEPC.

The magnitude of this error can be estimated. Assuming a time to peak of $0.9 \mathrm{msec}$ (a typical value), we can extrapolate the fast and slow exponential curves back to the time of MEPC onset and obtain a new estimate of the relative number of slow channel opcnings that includes events that occurred during the rising phase. This correction reduces an estimate of $30 \%$ slow channel openings to $18 \%$ slow, and an estimate of $80 \%$ slow becomes $67 \%$ slow. This brings the MEPC results into closer agreement with the spectral results. This is a rather simplistic correction, however, for it is based on the unrealistic assumption that all channel openings occur instantaneously at the time of MEPC onset. Therefore, we normally did not use this correction and simply kept in mind the inherent bias toward overestimating slow channel activity.

There may be other factors contributing to this discrepancy. For example, the desensitization kinetics of slow and fast channels may differ so that the brief, concentrated pulse of ACh that generates an MEPC opens relatively more slow channels than the prolonged, dilute ACh application used in noise recordings. In addition, it may be that our assumption that no channels open during the MEPC falling phase is incorrect. It is thought that, at most endplates, few ACh-sensitive channels open after the MEPC peak because synaptic acetylcholinesterase rapidly hydrolyzes any free transmitter in the cleft (Magleby and Stevens, 1972; Katz and Miledi, 1973). If the enzyme activity at soleus endplates were relatively low, however, some ACh might persist in the cleft after the MEPC peak, thus slowing the MEPC decay and leading to an overestimate of slow channel activity. We do not know to what extent these potential complicating factors atfected our analyses. Whatever the origins of the quantitative difference between MEPCs and noise spectra, the good qualitative agreement between these techniques indicates that MEPCs are adequate to judge the approximate maturity of an endplate with respect to channel conversion.

In interpreting the results of either technique, it is important to note that the estimates refer to the relative amount of fast and slow channel activity and not to the relative number of fast and slow AChR molecules present in the membrane. Thus, for example, if the 


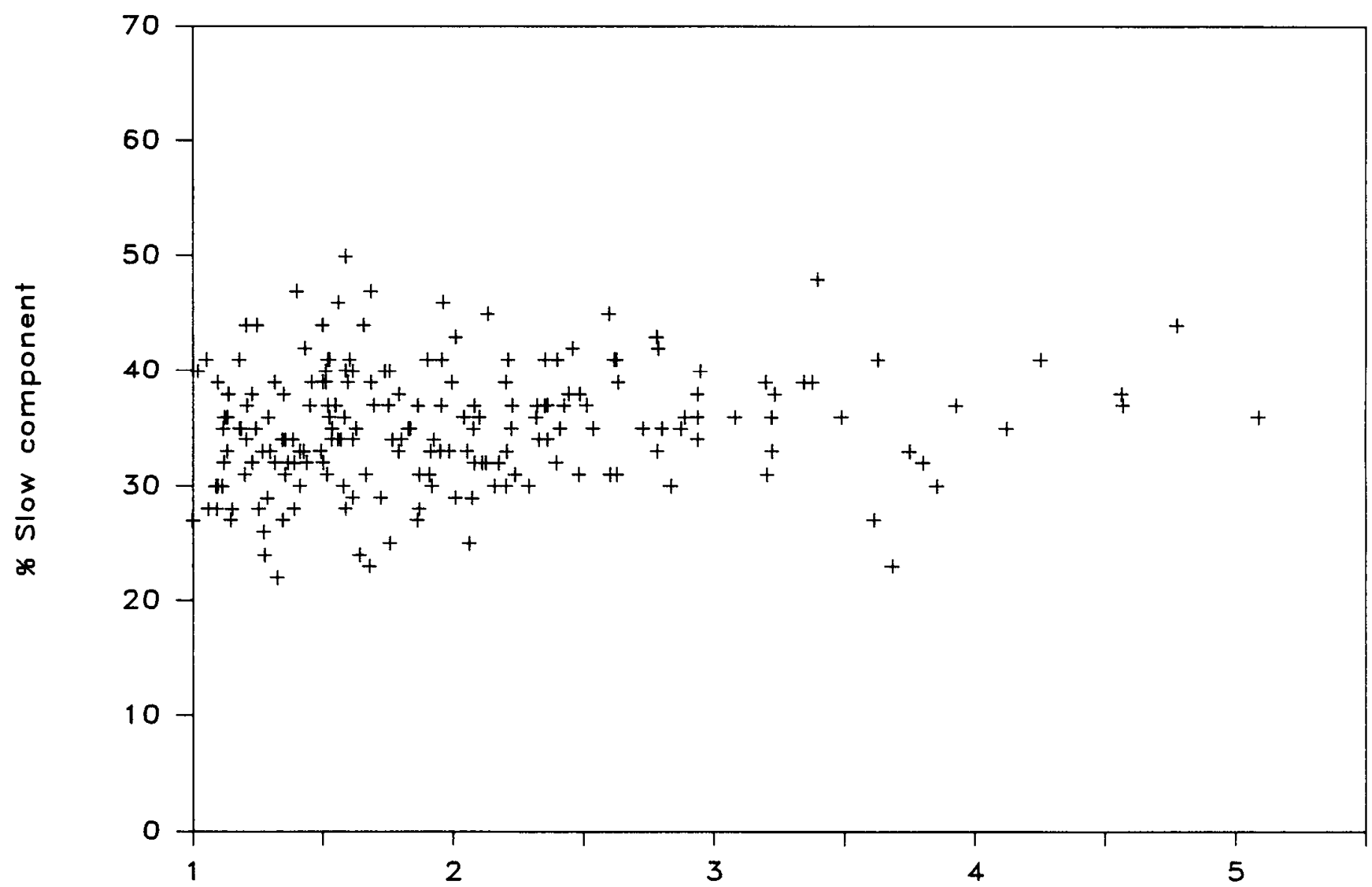

\section{Relative MEPC amplitude}

Figure 8. The relative size of the slow MEPC component plotted as a function of the relative peak amplitude of the entire MEPC. Each symbol represents one of 203 MEPCs recorded from a single endplate (same data set as in Fig. 7). Relative MEPC amplitude refers to peak MEPC height expressed as a multiple of the smallest MEPC in the series.

ACh noise spectrum at a particular endplate indicates $50 \%$ slow channel activity, this does not necessarily mean that the endplate contains equal numbers of embryonic-like and adult-like AChRs. The two types of AChRs may differ in their affinity for AChR or in their opening rates, either of which would affect the estimates of receptor activity.

Time course of channel conversion. It was clear that some fast channels could be detected as soon as 2 days after birth and that some slow channels persisted for at least 3 weeks after birth. Therefore, channel conversion occurs over a longer period than has been reported previously (Sakmann and Brenner, 1978; Fischbach and Schuetze, 1980). This probably reflects the increased sensitivity of the recording and analysis procedures. Although the relative amounts of fast and slow channel activity changed markedly with age, the estimated fast and slow $\tau$ 's remained relatively constant, unlike the developmental decreases in the $\tau$ 's reported for Xenopus myocytes in tissue culture (Leonard et al., 1983).

In agreement with earlier results (Fischbach and Schuetze, 1980), the relative amount of slow channel activity varied considerably from fiber to fiber within individual muscles. Thus, it appears that different fibers undergo channel conversion independently of each other. Because of this asynchrony, it is difficult to estimate how long channel conversion takes at individual endplates. At soleus endplates maintained in vitro, channel conversion apparently can occur quite rapidly, although the process is often interrupted by pauses of $1 \mathrm{hr}$ or more (S. M. Schuetze and S. Vicini, manuscript in preparation).

Variability among MEPCs within individual endplates. The relative amplitudes of the fast and slow exponential components varied from one MEPC to the next even at individual endplates. Initially, we hypothesized that there was variability in the relative activity of fast and slow channels within different endplate microregions, but we found no evidence to support this idea. Further consideration showed that most of the variability in MEPC decay phases could be explained more simply. Based on the results of simulation experiments, it appears that about $75 \%$ of the variance was due to a combination of (1) noise superimposed on the MEPCs by the recording apparatus, (2) the inherently random duration of channel openings, and (3) variability in the numbers of fast and slow channels activated due to the random binding of $\mathrm{ACh}$ to different members in the available pool of receptors.

Although we did not identify the factors that underlie the other $25 \%$ of the variance in the estimates of percentage of slow channel activity, there are several obvious possibilities. For example, we did not take into account variability in single-channel conductance and in $\tau$ among different channels of a given class. We also did not consider the possibility that some channels may open after the peak of the MEPC. These additional factors could well explain the remainder of the variance.

Postsynaptic folds at physiologically characterized endplates. The relative amounts of fast and slow AChR channel activity appear to be unrelated to the degree of postsynaptic membrane folding. Even though both processes take place during the same postnatal period, an endplate can have a substantial level of fast channel activity and virtually no secondary membrane folds. Although we did not make a detailed comparison, we found no other obvious morphological distinctions between endplates that differed in their rela- 

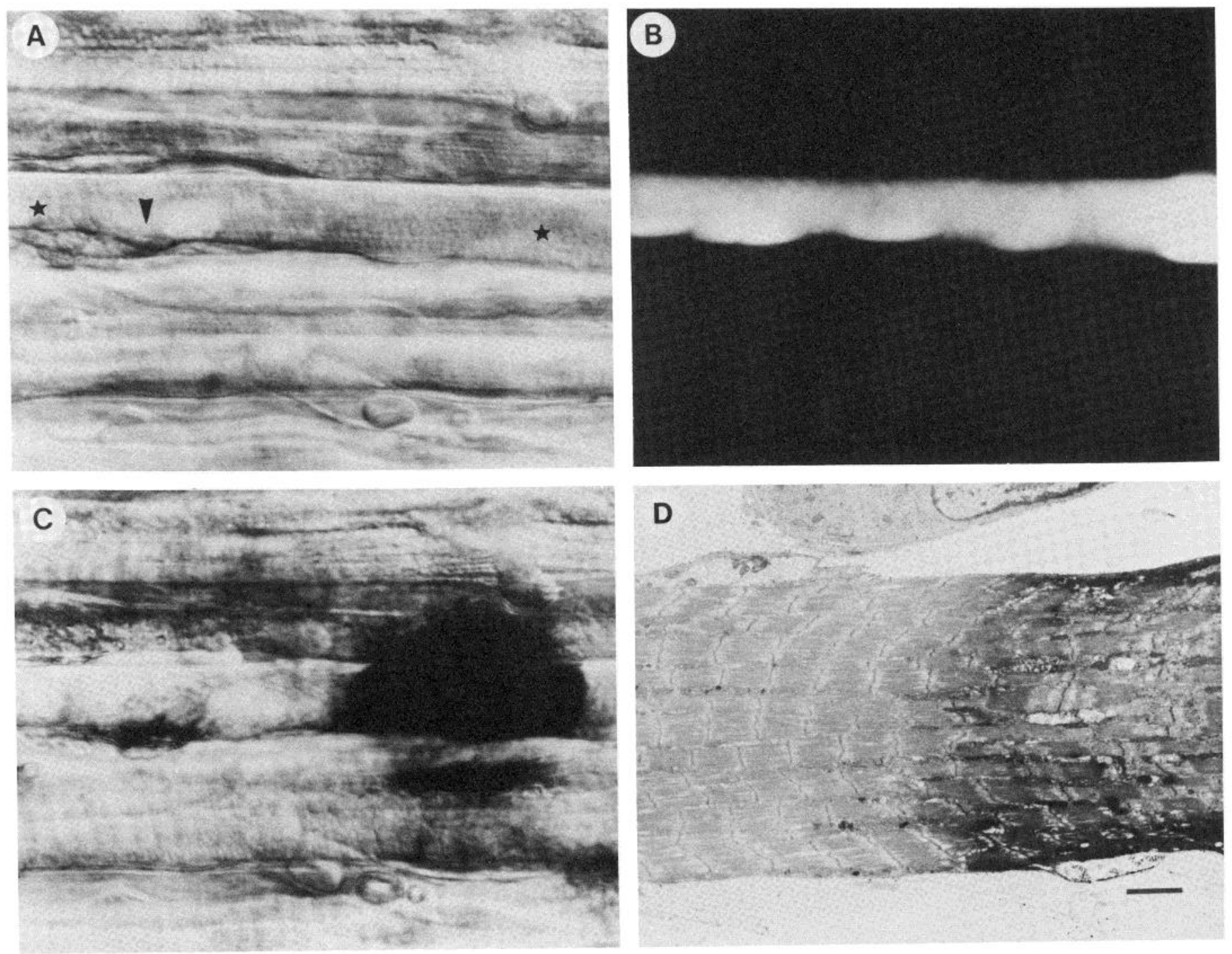

Figure 9. Labeling a muscle fiber with an electron-dense marker. A, Part of a living day 11 soleus muscle viewed with differential interference contrast optics. The stars mark the fiber that is to be labeled. The arrowhead points to its endplate region. $B$, The same field viewed with epi-illumination fluorescence optics after the fiber was injected with Lucifer Yellow. C. The same field after the cell was fixed, labeled with diaminobenzidine, and stained for acetylcholinesterase activity. $D$, The labeled fiber viewed in the transmission electron microscope. Note the transition between the labeled and unlabeled portions of the fiber. Bar $=10 \mu \mathrm{m}(A$ to $C)$ or $2.3 \mu \mathrm{m}(D)$.

tive levels of slow channel activity, except to the extent that both physiological and morphological maturity increased with age. However, our micrographs were relatively low power (approximately $x$ 5,000 to 20,000 ), and there may have been other structural differences that would have been apparent only at higher magnification.

Other evidence that channel conversion and synaptic folds are not related comes from the observation that AChRs in extrajunctional regions of developing innervated fibers may have predominantly fast channel activity despite their lack of folds (Schuetze and Vicini, 1984). In innervated cells in which the level of extrajunctional ACh sensitivity was high enough for noise analysis, AChR channel properties were identical at synaptic and extrasynaptic sites on the same cells. Similar conclusions have been drawn from studies of Xenopus muscle (Kullberg et al., 1981; Brehm et al., 1984).

Finally, the coexistence of fast and slow channels within the same small patch of membrane, as shown by patch clamp recordings (Figs. 3 and 6), suggests that AChR gating properties are not governed primarily by the channel's membrane environment. In fact, recent work indicates that embryonic-type and adult-type AChR channels differ in their molecular structure (Schuetze et al., 1985).

The conductance of fast and slow soleus AChRs. In agreement with earlier studies of rat myotubes in tissue culture, single-channel

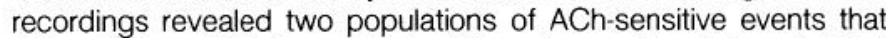
differed about $50 \%$ in amplitude. Although we did not do a detailed analysis of our records, it was clear that the higher conductance channels were fast and the lower conductance channels were slow. We did not notice any conductance change with age for either type of AChR.

In contrast to other reports showing ohmic behavior of AChsensitive channels in rat myotubes (Siegelbaum et al., 1984), channels in soleus muscles appeared to rectify. At least in part, this was probably due to our use of L-15 in the patch pipette in early experiments. Arginine in the L-15 medium would be expected to generate such rectification (Sanchez et al., 1983). The rectification was less pronounced or even absent when the patch pipette contained rat Ringer rather than L-15, but we did not do enough experiments for the results to be conclusive. Brehm et al. (1984) also reported rectification in ACh-sensitive channels in Xenopus myocytes, but in their experiments the conductance increased, rather than decreased, with hyperpolarization.

Summary. These experiments demonstrate that MEPC analysis is a simple, rapid procedure for estimating the relative levels of embry- 


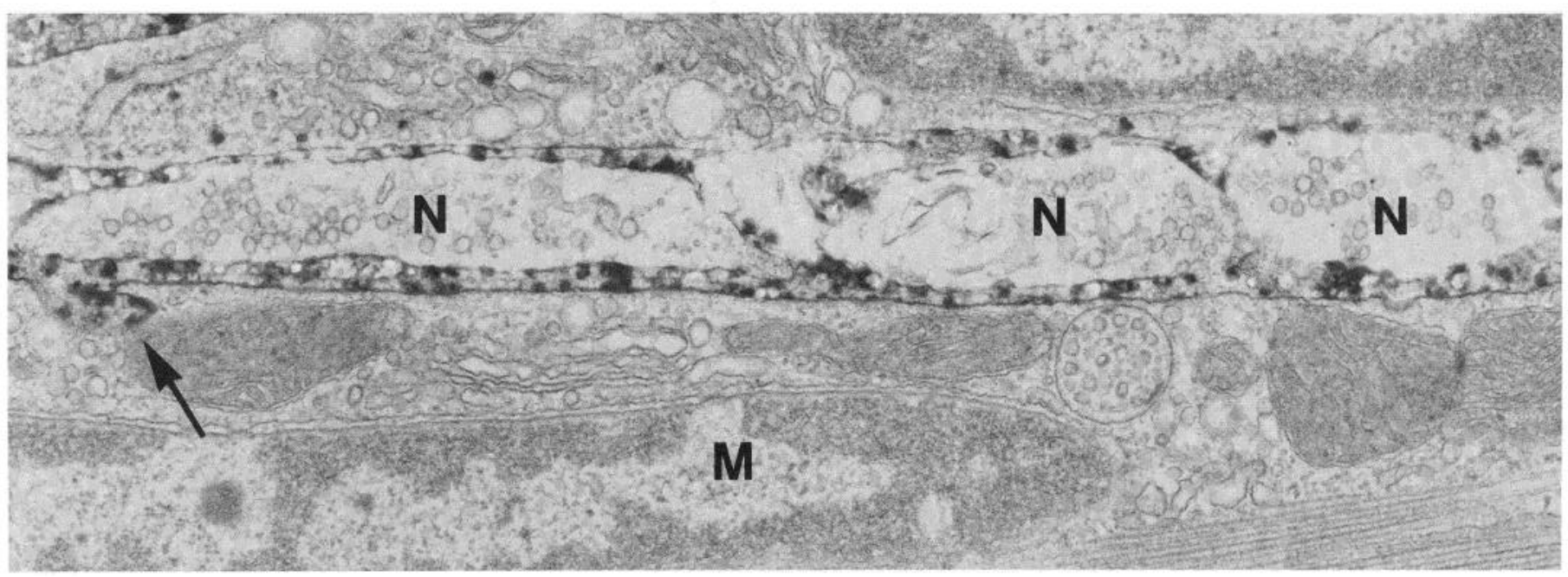

Figure 10. Electron micrograph of a day 8 endplate that had been studied physiologically. MEPCs at this endplate were doubly exponential and the fast component was $41 \%$ of peak MEPC amplitude, which indicated that at least $32 \%$ of the channel activity was due to fast, adult-like AChRs. The cell was then labeled as described in Figure 9, embedded, semi-serially sectioned, and examined in the electron microscope. This photograph shows the region with maximum membrane folding (arrow). The dark granular material is the reaction product of the histochemical stain for cholinesterase activity. $N$, nerve terminal; $M$, muscle. Magnification $\times 27,000$.

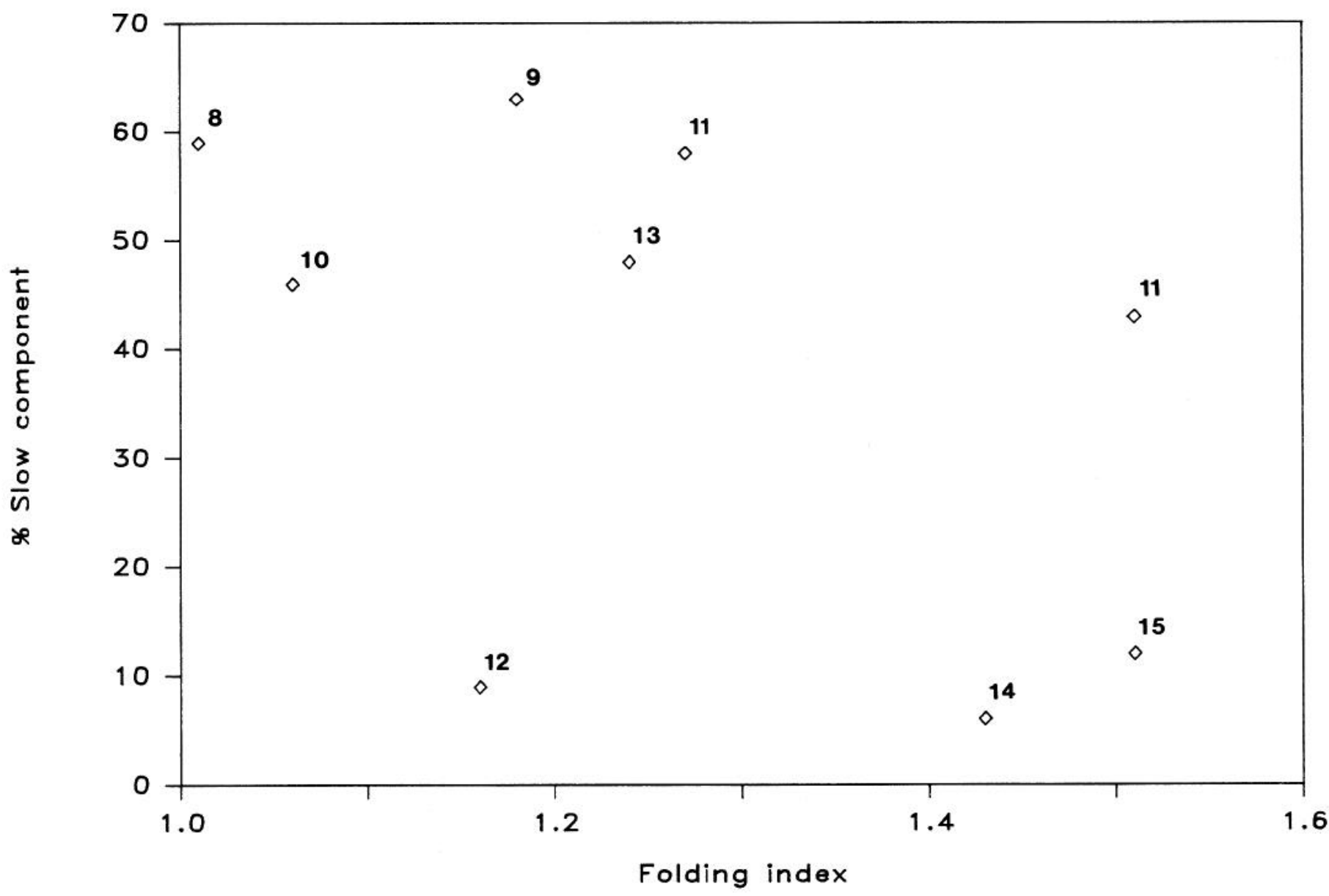

Figure 11. Lack of correlation between the relative size of the slow MEPC component and membrane folding. MEPCs were recorded and analyzed from one endplate in each of nine different soleus muscles. Each fiber was labeled and examined in the electron miscroscope. The extent of membrane folding was characterized by computing a folding index, defined as the ratio of the total length of the endplate membrane (including folds) to the length of the endplate through the midline of the synaptic cleft (ignoring folds). The number by each symbol represents the age of the rat (days). 
onic-like and adult-like AChR activity at developing endplates. Results obtained with this technique indicate that channel conversion occurs within different regions of individual endplates more or less synchronously. However, different endplates within the same muscle change their channel properties asynchronously. The developmental changes in channel properties apparently are completely unrelated to the formation of postsynaptic folds or to any other obvious morphological specializations.

\section{References}

Anderson, C. R., and C. F. Stevens (1973) Voltage clamp analysis of acetylcholine produced endplate current fluctuations at frog neuromuscular junction. J. Physiol. (Lond.) 235: 655-691.

Bevan, S., and J. H. Steinbach (1977)The distribution of $\alpha$-bungarotoxin binding sites on mammalian skeletal muscle developing in vivo. J. Physiol. (Lond.) 267: 195-213.

Brehm, P., R. Kullberg, and F. Moody-Corbett (1984) Properties of nonjunctional acetylcholine receptor channels on innervated muscle of Xenopus laevis. J. Physiol. (Lond.) 350: 631-648.

Brenner, H. R., and B. Sakmann (1983) Neurotrophic control of channel properties at neuromuscular synapses of rat muscle. J. Physiol. (Lond.) 337: 159-171.

Brockes, J., and Z. W. Hall (1975) Acetylcholine receptors in normal and denervated rat diaphragm muscle. II. Comparison of junctional and extrajunctional receptors. Biochemistry 14: 2100-2106.

Colquhoun, D., and B. Sakmann (1981) Fluctuations in the microsecond time range of the current through single acetylcholine receptor ion channels. Nature 294: 464-466.

Dennis, M. J., L. Ziskind-Conhaim, and A. J. Harris (1981) Development of neuromuscular junctions in rat embryos. Dev. Biol. 81: 266-279.

Fambrough, D. M. (1979) Control of acetylcholine receptors in skelctal muscle. Physiol. Rev. 59: 165-227.

Fischbach, G. D., and S. M. Schuetze (1980) A postnatal decrease in acetylcholine channel open time at rat endplates. J. Physiol. (Lond.) 303: 125-137.

Frank, E., and G. D. Fischbach (1979) Early events in neuromuscular junction formation in vitro: Induction of acetylcholine receptor clusters in the postsynaptic membrane and morphology of newly formed nerve-muscle synapses. J. Cell Biol. 83: 143-158.

Hall, Z. W., M.-P. Roisin, Y. Gu, and P. D. Gorin (1984) A developmental change in the immunological properties of acetylcholine receptors at the rat neuromuscular junction. Cold Spring Harhor Symp. Quant. Biol. 47: 101-108.

Hamill, O. P., and B. Sakmann (1981) Multiple conductance states of single acctylcholine receptor channcls in cmbryonic muscle cells. Nature 294: $462-464$.

Hamill, O. P., A. Marty, E. Neher, B. Sakmann, and F. J. Sigworth (1981) In proved patch-clamp lechmiques for hight-resolution current recording from cells and cell-free membrane patches. Pflugers Arch. 391:85-100.

Katz, B., and R. Miledi (1973) The binding of acetylcholine to receptors and its removal trom the synaptic clett. J. Physiol. (Lond.) 231: 549-5/4.
Korneliussen, H., and J. K. S. Jansen (1976) Morphological aspects of the elimination of polyneuronal innervation of skeletal muscle of newborn rats. J. Neurocytol. 5: 591-604.

Kullberg, R. W., P. Brehm, and J. H. Steinbach (1981) Nonjunctional acetyl choline receptor channel open time decreases during development of Xenopus muscle. Nature 289: 411-413.

Leonard, R. J., Y. Nakajima, S. Nakajima, and T. Takahashi (1983) Single channel open time of two types of ACh receptors in cultured Xenopus myocytes decrease during development. Soc. Neurosci. Abstr. 9: 1180.

Magleby, K. L., and C. F. Stevens (1972) A quantitative description of endplate currents. J. Physiol. (Lond.) 223: 173-197.

Maranto, A. R. (1982) Neuronal mapping: A photooxidation reaction makes Lucifer Yellow useful for electron microscopy. Science 217: 953-955.

Matthews-Bellinger, J. A., and M. M. Salpeter (1983) Fine structural distribution of acetylcholine receptors at developing mouse neuromuscular junctions. J. Neurosci. 3: 644-657.

Michler, A., and B. Sakmann (1980) Receptor stability and channel conversion in the subsynaptic membrane of the developing mammalian neuromuscular junction. Dev. Biol. 80: 1-17.

Reiness, C. G., and Z. W. Hall (1981) The developmental change in immunological properties of the acetylcholine receptor in rat muscle. Dev. Biol. 81: $324-331$.

Reiness, C. G., and C. B. Weinberg (1981) Metabolic stabilization of acetylcholine receptors at newly formed neuromuscular junctions in the rat. Dev. Biol. 84: 247-254.

Rosenthal, J. L., and P. S. Taraskevich (1977) Reduction of multiaxonal innervation at the neuromuscular junction of the rat during development. J. Physiol. (Lond.) 270: 299-310.

Sakmann, B., and H. R. Brenner (1978) Change in synaptic channel gating during neuromuscular development. Nature 276: 401-402.

Sanchez, J. A., J. A. Dani, D. Siemen, and B. Hille (1983) Block and possible agonist action of permeant organic ions at cholinergic channels. Biophys. J. $41: 65 a$

Schuetze, S. M. (1980) The acetylcholine channel open time in chick muscle is not decreased following innervation. J. Physiol. (Lond.) 303: 111-124.

Schuetze, S. M., and G. D. Fischbach (1978) Channel open time decreases postnatally in rat synaptic acetylcholine receptors. Soc. Neurosci. Abstr. 4: 374 .

Schuetze, S. M., and S. Vicini. (1984) Neonatal denervation inhibits the normal postnatal decrease in endplate channel open time. J. Neurosci. 4 : 2297-2302.

Schuetze, S. M., S. Vicini, and Z. W. Hall (1985) A myasthenic serum selectively blocks acetylcholine receptors with long channel open times at developing rat endplates. Proc. Natl. Acad. Sci. U. S. A. 82: 2533-2537.

Siegelbaum, S. A., A. Trautmann, and J. Koenig (1984) Single acetylcholineactivated channel currents in developing muscle cells. Dev. Biol. 104: 366-379.

Steinbach, J. H. (1981) Developmental changes in acetylcholine receptor aggregates at rat skeletal neuromuscular junctions. Dev. Biol. 84: $267-$ 276.

Ziskind, L., and M. J. Dennis (1978) Depolarising effect of curare on embryonic rat muscles. Nature 2/6: 622-623. 